\title{
On the $\boldsymbol{k}$-Lucas Numbers and Lucas Polynomials
}

\author{
Ali Boussayoud ${ }^{1, *}$, Mohamed Kerada ${ }^{1}$, Nesrine Harrouche ${ }^{2}$ \\ ${ }^{1}$ LMAM Laboratory and Department of Mathematics, Mohamed Seddik Ben Yahia University, Jijel, Algeria \\ ${ }^{2}$ Department of Mathematics, University of Jordan, Amman 11942, Jordan \\ *Corresponding author: aboussayoud@yahoo.fr
}

Received December 23, 2016; Revised May 11, 2017; Accepted June 07, 2017

\begin{abstract}
In this paper, we introduce an operator in order to derive some new symmetric properties of $k$-Lucas numbers and Lucas polynomials. By making use of the operator defined in this paper, we give some new generating functions for $k$-Lucas numbers and Lucas polynomials.
\end{abstract}

Keywords: k-Lucas numbers, Lucas polynomials, generating functions

Cite This Article: Ali Boussayoud, Mohamed Kerada, and Nesrine Harrouche, "On the $k$-Lucas Numbers and Lucas Polynomials.” Turkish Journal of Analysis and Number Theory, vol. 5, no. 4 (2017): 121-125. doi: 10.12691/tjant-5-4-1.

\section{Introduction}

Fibonacci and Lucas numbers have been studied by many researchers for a long time to get intrinsic theory and applications of these numbers in many research areas as Physics, Engineering, Architecture, Nature and Art. For example, the ratio of two consecutive numbers converges to the Golden ratio $\alpha=\frac{1+\sqrt{5}}{2}$ which was thoroughly interested in [13]. We should recall that, for $k \in \mathbb{R}_{+}$, $k$-Fibonacci $\quad\left\{F_{\mathrm{k}, \mathrm{n}}\right\}_{n \in \mathbb{N}}$ and $k$-Lucas $\left\{L_{k, n}\right\}_{n \in \mathbb{N}}$ sequences have been defined by the recursive equations $[9,10]$;

$$
\begin{aligned}
& F_{k, n+2}=k F_{k, n+1}+F_{k, n}, \\
& L_{k, n+2}=k L_{k, n+1}+L_{k, n},
\end{aligned}
$$

with initial conditions $F_{k, 0}=1, \quad F_{k, 1}=k$ and $L_{k, 0}=2$, $L_{k, 1}=k$, respectively. For the special case $k=1$, it is clear that these two sequences are simplified to the well-known Fibonacci and Lucas sequences, respectively. In this contribution, we shall define a new useful operator denoted by $\delta_{p_{1} p_{2}}^{k}$ for which we can formulate, extend and prove new results based on our previous ones $[4,5,6]$. In order to determine generating functions for $k$-Fibonacci numbers, $k$-Lucas numbers and Lucas polynomials, we combine between our indicated past techniques and these presented polishing approaches.

Let $k$ and $n$ be two positive integers and $\left\{x_{1}, x_{2}, \ldots, x_{n}\right\}$ are set of given variables, recall [8] that the $k$-th elementary symmetric function $e_{k}\left(x_{1}, x_{2}, \ldots, x_{n}\right)$ and the $k$-th complete homogeneous symmetric function $h_{k}\left(x_{1}, x_{2}, \ldots, x_{n}\right)$ are defined respectively by $e_{k}\left(x_{1}, x_{2}, \ldots, x_{n}\right)=\sum_{i_{1}+i_{2}+\ldots+i_{n}=k} x_{1}^{i_{1}} x_{2}^{i_{2}} \ldots x_{n}^{i_{n}}, 0 \leq k \leq n$.

With $i_{1}, i_{2}, \ldots, i_{n}=0$ or 1 ,

$h_{k}\left(x_{1}, x_{2}, \ldots, x_{n}\right)=\sum_{i_{1}+i_{2}+\ldots+i_{n}=k} x_{1}^{i_{1}} x_{2}^{i_{2}} \ldots x_{n}^{i_{n}}, 0 \leq k \leq n$.

With $i_{1}, i_{2}, \ldots, i_{n} \geq 0$,

First, we set $e_{0}\left(x_{1}, x_{2}, \ldots, x_{n}\right)=1$ and $h_{0}\left(x_{1}, x_{2}, \ldots, x_{n}\right)$

$=1$ (by convention).

For $k>n$ or $k<0$, we set

$$
e_{k}\left(x_{1}, x_{2}, \ldots, x_{n}\right)=0 \text { and } h_{k}\left(x_{1}, x_{2}, \ldots, x_{n}\right)=0 \text {. }
$$

Definition 1. [1] Let $B$ and $P$ be any two alphabets, then we give $S_{n}(B-P)$ by the following form:

$$
\frac{\prod_{p \in P}(1-p t)}{\prod_{b \in B}(1-b t)}=\sum_{n=0}^{\infty} S_{n}(B-P) t^{n},
$$

with the condition $S_{n}(B-P)=0$ for $n<0$.

Definition 2. [2] Let $g$ be any function on $\mathrm{R}^{n}$, then we consider the divided difference operator as the following form

$$
\partial_{x_{i}, x_{i+1}}(g)=\frac{\left[\begin{array}{l}
g\left(x_{1}, \ldots, x_{i}, x_{i+1}, \ldots, x_{n}\right) \\
-g\left(x_{1}, \ldots x_{i-1}, x_{i+1}, x_{i}, \ldots, x_{n}\right)
\end{array}\right]}{x_{i}-x_{i+1}} .
$$

Definition 3. [7] The symmetrizing operator $\delta_{p_{1} p_{2}}^{k}$ is defined by

$$
\delta_{p_{1} p_{2}}^{k} g\left(p_{1}\right)=\frac{p_{1}^{k} g\left(p_{1}\right)-p_{2}^{k} g\left(p_{2}\right)}{p_{1}-p_{2}} \text { for all } k \in \mathbb{N} .
$$

Remark 1. Let $P=\left\{p_{1}, p_{2}\right\}$ an alphabet, we have 


$$
h_{k}\left(p_{1}, p_{2}\right)=S_{k}\left(p_{1}+p_{2}\right)=\delta_{p_{1} p_{2}}^{k}\left(p_{1}\right) .
$$

\section{The $k$-Lucas Numbers and Properties}

The $k$-Lucas numbers have been defined in [11] for any number $k$ as follows.

Definition 4. [11] For any positive real number, the $k$-Lucas numbers, say $\left\{L_{k, n}\right\}_{n \in \mathbb{N}}$ is defined recurrently by

$$
L_{k, n+1}=k L_{k, n}+L_{k, n-1} \text { for } n \geq 1 \text {, }
$$

with initial conditions $L_{k, 0}=2, L_{k, 1}=k$.

Note that if $k$ is a real variable $x$ then $L_{k, n}=L_{x, n}$ and they correspond to the Lucas polynomials defined by

$$
L_{n+1}(x)= \begin{cases}2 & \text { if } n=0 \\ x & \text { if } n=1 \\ x L_{n}(x)+L_{n-1}(x) & \text { if } n>1\end{cases}
$$

Particular cases of the $k$-Lucas numbers are

- If $k=1$, the classical Lucas numbers is obtained:

$$
\begin{aligned}
& L_{0}=2, L_{1}=1, \text { and } \\
& L_{n+1}=L_{n}+L_{n-1} \text { for } n \geq 1: \\
& \left\{L_{n}\right\}_{n \in \mathbb{N}}=\{2,1,3,4,7, \ldots\} .
\end{aligned}
$$

- If $k=2$, the Pell-Lucas numbers appears:

$$
\begin{aligned}
& Q_{0}=2, Q_{1}=2, \text { and } \\
& Q_{n+1}=2 Q_{n}+Q_{n-1} \text { for } n \geq 1 \\
& \left\{Q_{n}\right\}_{n \in \mathbb{N}}=\{2,2,6,14,34, \ldots\} .
\end{aligned}
$$

The well-known Binet's formula in the Lucas numbers theory allows us to express the $k$-Lucas number in function of the roots $r_{1}$ and $r_{2}$ of the characteristic equation, associated to the recurrence relation (2.1):

$$
r^{2}=k r+1
$$

Proposition 1. (Binet's formula) The nth $k$-Lucas number is given by

$$
L_{k, n}=\frac{r_{1}^{n}-r_{2}^{n}}{r_{1}-r_{2}}
$$

where $r_{1}, r_{2}$ are the roots of the characteristic equation (2.2) and $r_{1}>r_{2}$.

Proof . The roots of the characteristic equation (2.2) are $r_{1}=\frac{k+\sqrt{k^{2}+4}}{2}$ and $r_{2}=\frac{k-\sqrt{k^{2}+4}}{2}$.

Note that, since $k>0$, the $r_{2}<0<r_{1}$ and $\left|r_{2}\right|<\left|r_{1}\right|$, $r_{1}+r_{2}=k$ and $r_{1} \cdot r_{2}=-1, r_{1}-r_{2}=\sqrt{k^{2}+4}$.

If $\sigma$ denotes the positive root of the characteristic equation, the general term may be written in the form [10]

$$
L_{k, n}=\frac{\sigma^{n}-\sigma^{-n}}{\sigma+\sigma^{-1}}
$$

and the limit of the quotient of two terms is

$$
\lim _{n \rightarrow \infty} \frac{L_{k, n+r}}{L_{k, n}}=\sigma^{r} .
$$

In addition, the general term of the $k$-Lucas numbers may be obtained by the formula [10]:

$$
L_{k, n}=k F_{k, n-1}+F_{k, n+1} \text {. }
$$

\section{On the Symmetric Functions of Some Numbers and Polynomails}

Theorem 1. [4] Let $P$ and $B$ be two alphabets, respectively, $\left\{p_{1}, p_{2}\right\}$ and $\left\{b_{1}, b_{2}, b_{3}\right\}$, then we have

$$
\begin{aligned}
& \sum_{n=0}^{\infty} h_{n}\left(b_{1}, b_{2}, b_{3}\right) h_{n}\left(p_{1}, p_{2}\right) t^{n} \\
& =\frac{\left(\begin{array}{l}
1-p_{1} p_{2} e_{2}\left(b_{1}, b_{2}, b_{3}\right) t^{2} \\
-p_{1} p_{2} e_{2}\left(b_{1}, b_{2}, b_{3}\right) h_{1}\left(p_{1}, p_{2}\right) t^{3}
\end{array}\right)}{\left(\sum_{n=0}^{\infty} e_{n}\left(b_{1}, b_{2}, b_{3}\right) p_{1}^{n} t^{n}\right)\left(\sum_{n=0}^{\infty} e_{n}\left(b_{1}, b_{2}, b_{3}\right) p_{2}^{n} t^{n}\right)} .
\end{aligned}
$$

In the case $B=\{1\}$ based on Theorem 1, we deduce the following Lemmas.

Lemma 1. Given an alphabet $P=\left\{p_{1},-p_{2}\right\}$, we have

$$
\sum_{n=0}^{\infty} h_{n}\left(p_{1},\left[-p_{2}\right]\right) t^{n}=\frac{1}{1-\left(p_{1}-p_{2}\right) t-p_{1} p_{2} t^{2}}
$$

Proof. Let $\sum_{n \geq 0}^{\infty} p_{1} t^{n}$ and $\left(1-p_{1} t\right)$ be two sequences such that $\sum_{n \geq 0}^{\infty} p_{1}^{n} t^{n}=\frac{1}{1-p_{1} t}$, the left-hand side of the formula (3.2) can be written as:

$$
\begin{aligned}
\delta_{p_{1}\left[-p_{2}\right]} \sum_{n=0}^{\infty} p_{1}^{n} t^{n} & =\frac{p_{1} \sum_{n=0}^{\infty} p_{1}^{n} t^{n}-p_{2} \sum_{n=0}^{\infty}\left[-p_{2}\right]^{n} t^{n}}{p_{1}-\left[-p_{2}\right]} \\
& =\sum_{n=0}^{\infty} \frac{p_{1}^{n+1}-\left[-p_{2}\right]^{n+1}}{p_{1}-\left[-p_{2}\right]} t^{n} \\
& =\sum_{n=0}^{\infty} h_{n}\left(p_{1},\left[-p_{2}\right]\right) t^{n} .
\end{aligned}
$$

white the right -hand side can be expressed as

$$
\begin{aligned}
\delta_{p_{1}\left[-p_{2}\right] \frac{1}{1-p_{1} t}} & =\frac{p_{1} \frac{1}{1-p_{1} t}-\left[-p_{2}\right] \frac{1}{1-\left[-p_{2}\right] t}}{p_{1}-\left[-p_{2}\right]} \\
& =\frac{p_{1} \frac{1}{1-p_{1} t}-\left[-p_{2}\right] \frac{1}{1+p_{2} t}}{p_{1}-\left[-p_{2}\right]} \\
& =\frac{p_{1}\left(1+p_{2} t\right)+p_{2}\left(1-p_{1} t\right)}{\left(p_{1}+p_{2}\right)\left(1-p_{1} t\right)\left(1+p_{2} t\right)} \\
& =\frac{p_{1}+p_{2}}{\left(p_{1}+p_{2}\right)\left(1-\left(p_{1}-p_{2}\right) t-p_{1} p_{2} t^{2}\right)} \\
& =\frac{1}{1-\left(p_{1}-p_{2}\right) t-p_{1} p_{2} t^{2}}
\end{aligned}
$$


This completes the proof.

Lemma 2. Given an alphabet $P=\left\{p_{1},-p_{2}\right\}$, we have

$$
\sum_{n=0}^{\infty} h_{n+1}\left(p_{1},\left[-p_{2}\right]\right) t^{n}=\frac{p_{1}-p_{2}+p_{1} p_{2} t}{1-\left(p_{1}-p_{2}\right) t-p_{1} p_{2} t^{2}}
$$

Proof. Let $\sum_{n \geq 0}^{\infty} p_{1} t^{n}$ and $\left(1-p_{1} t\right)$ be two sequences such that $\sum_{n \geq 0}^{\infty} p_{1}^{n} t^{n}=\frac{1}{1-p_{1} t}$, the left-hand side of the formula (3.3) can be written as:

$$
\begin{aligned}
\delta_{p_{1}\left[-p_{2}\right]}^{2} \sum_{n=0}^{\infty} p_{1}^{n} t^{n}= & \frac{p_{1}^{2} \sum_{n=0}^{\infty} p_{1}^{n} t^{n}}{p_{1}-\left[-p_{2}\right]} \\
& -\frac{\left[-p_{2}\right]^{2} \sum_{n=0}^{\infty}\left[-p_{2}\right]^{n} t^{n}}{p_{1}-\left[-p_{2}\right]} \\
& =\sum_{n=0}^{\infty} \frac{p_{1}^{n+2}-\left[-p_{2}\right]^{n+2}}{p_{1}-\left[-p_{2}\right]} t^{n} \\
& =\sum_{n=0}^{\infty} h_{n+1}\left(p_{1},\left[-p_{2}\right]\right) t^{n} .
\end{aligned}
$$

white the right-hand side can be expressed as:

$$
\begin{aligned}
\delta_{p_{1}\left[-p_{2}\right] \frac{1}{1-p_{1} t}}^{2} & \frac{p_{1}^{2} \frac{1}{1-p_{1} t}-\left[-p_{2}\right]^{2} \frac{1}{1-\left[-p_{2}\right] t}}{p_{1}-\left[-p_{2}\right]} \\
& =\frac{p_{1}^{2}\left(1+p_{2} t\right)-p_{2}^{2}\left(1-p_{1} t\right)}{\left(p_{1}+p_{2}\right)\left(1-p_{1} t\right)\left(1+p_{2} t\right)} \\
& =\frac{p_{1}^{2}+p_{1}^{2} p_{2} t-p_{2}^{2}+p_{2}^{2} p_{1} t}{\left(p_{1}+p_{2}\right)\left(1-\left(p_{1}-p_{2}\right) t-p_{1} p_{2} t^{2}\right)} \\
& =\frac{p_{1}^{2}-p_{2}^{2}+p_{1} p_{2}\left(p_{1}+p_{2}\right) t}{\left(p_{1}+p_{2}\right)\left(1-\left(p_{1}-p_{2}\right) t-p_{1} p_{2} t^{2}\right)} \\
& =\frac{p_{1}-p_{2}+p_{1} p_{2} t}{1-\left(p_{1}-p_{2}\right) t-p_{1} p_{2} t^{2}} .
\end{aligned}
$$

This completes the proof.

Taking $p_{1}-p_{2}=1$ and $p_{1} p_{2}=1$ in (3.2) and (3.3), we obtain the generating functions given by Boussayoud et al [5] which arises

1) The generating function of the Fibonacci numbers

$$
\sum_{n=0}^{\infty} F_{n} t^{n}=\frac{1}{1-t-t^{2}}
$$

2) The generating function of the Lucas numbers

$$
\sum_{n=0}^{\infty} L_{n} t^{n}=\frac{2-t}{1-t-t^{2}}
$$

Proposition 2. [10,12] The relations
1) $F_{k,-n}=(-1)^{n+1} F_{k, n}$,
2) $L_{k,-n}=(-1)^{n} L_{k, n}$ hold for all $n \geq 0$.

Choosing $p_{1}$ and $p_{2}$ such that $\left\{\begin{array}{c}p_{1} p_{2}=1 \\ p_{1}-p_{2}=k\end{array}\right.$ and substituting in (3.2) and (3.3) we end up with

$$
\sum_{n=0}^{\infty} h_{n}\left(p_{1},\left[-p_{2}\right]\right) t^{n}=\sum_{n=0}^{\infty} F_{k, n} t^{n}=\frac{1}{1-k t-t^{2}},
$$

which represents a generatings functions for $k$-Fibonacci numbers (with $p_{1}=r_{1}$ and $\left[-p_{2}\right]=r_{2}$ ).

$$
\sum_{n=0}^{\infty} h_{n+1}\left(p_{1},\left[-p_{2}\right]\right) t^{n}=\frac{k+t}{1-k t-k t^{2}},
$$

which represents a new generatings functions.

- Multiplying the equation (3.4) by $\left(2+k^{2}\right)$ and subtract it from (3.5) by $(k)$, we obtain

$$
\begin{aligned}
& \sum_{n=0}^{\infty}\left[\left(2+k^{2}\right) h_{n}\left(p_{1},\left[-p_{2}\right]\right)-k h_{n+1}\left(p_{1},\left[-p_{2}\right]\right)\right] t^{n} \\
& =\frac{2-k t}{1-k t-t^{2}},
\end{aligned}
$$

from which we have the following theorem.

Theorem 3. For $n \in \mathbb{N}$, the generating function of the $k$-Lucas numbers is given by

$$
\sum_{n=0}^{\infty} L_{k, n} t^{n}=\frac{2-k t}{1-k t-t^{2}}
$$

- Put $k=2$ in the relationship (3.6) we have

$$
\sum_{n=0}^{\infty} Q_{n} t^{n}=\frac{2-2 t}{1-2 t-t^{2}},
$$

which represents a generating function for Pell-Lucas numbers [5].

Replacing $t$ by $(-t)$ in (3.4) and (3.6), we have the following theorems.

Theorem 4. We have the following a new generating function of the $k$-Fibonacci numbers at negative indices as

$$
\sum_{n=0}^{\infty} F_{k,-n} t^{n}=\frac{1}{t^{2}-k t-1}
$$

Proof. The ordinary generating function associated is defined by

$$
G\left(F_{k, n}, t\right)=\sum_{n=0}^{\infty} F_{k, n} t^{n}
$$

Using the initial conditions, we get

$$
\begin{aligned}
\sum_{n=0}^{\infty} F_{k, n} t^{n} & =F_{k, 0} t^{0}+F_{k, 1} t+\sum_{n=2}^{\infty} F_{k, n} t^{n} \\
& =1+k t+\sum_{n=2}^{\infty} k F_{k, n-1} t^{n}+\sum_{n=2}^{\infty} F_{k, n-2} t^{n} .
\end{aligned}
$$

Consider that $j=n-2$ and $p=n-1$. Then can be written by

$$
\begin{aligned}
& =1+k t+t \sum_{n=1}^{\infty} k F_{k, n} t^{n}+t^{2} \sum_{j=0}^{\infty} F_{k, n} t^{n} \\
& =1+k t+k t \sum_{p=0}^{\infty} F_{k, n} t^{n}-k t+t^{2} \sum_{j=0}^{\infty} F_{k, n} t^{n},
\end{aligned}
$$


which is equivalent to

$$
\begin{aligned}
\left(1-k t-t^{2}\right) & \sum_{n=0}^{\infty} F_{k, n} t^{n}=1 \\
\Rightarrow \sum_{n=0}^{\infty} F_{k, n} t^{n} & =\frac{1}{1-k t-t^{2}} .
\end{aligned}
$$

Replacing $t$ by $(-t)$, we have

$$
\sum_{n=0}^{\infty}(-1)^{n} F_{k, n} t^{n}=\frac{1}{1+k t-t^{2}},
$$

therefore

$$
\begin{gathered}
\sum_{n=0}^{\infty}(-1)^{n+1} F_{k, n} t^{n}=\frac{1}{t^{2}-k t-1} \\
\Rightarrow \sum_{n=0}^{\infty} F_{k,-n} t^{n}=\frac{1}{t^{2}-k t-1} .
\end{gathered}
$$

This completes the proof.

Theorem 5. We have the following a new generating function of the $k$-Lucas numbers at negative indices as

$$
\sum_{n=0}^{\infty} L_{k,-n} t^{n}=\frac{2+k t}{1+k t-t^{2}} .
$$

- Put $k=2$ in the relationship (3.7) we have

$$
\sum_{n=0}^{\infty} Q_{-n} t^{n}=\frac{2+2 t}{1+2 t-t^{2}},
$$

which represents a generating function for Pell-Lucas numbers at negative indices [3].

Choosing $p_{1}$ and $p_{2}$ such that $\left\{\begin{array}{c}p_{1} p_{2}=1 \\ p_{1}-p_{2}=x\end{array}\right.$ and substituting in (3.2) and (3.3) we end up with

$$
\sum_{n=0}^{\infty} F_{n}(x) t^{n}=\frac{1}{1-x t-t^{2}},
$$

which represents a generating function of the Fibonacci polynomials

$$
\sum_{n=0}^{\infty} h_{n+1}\left(p_{1},\left[-p_{2}\right]\right) t^{n}=\frac{x+t}{1-x t-k t^{2}},
$$

which represents a new generatings functions.

- Multiplying the equation (3.8) by $\left(2+x^{2}\right)$ and subtract it from (3.9) by $(x)$, we obtain

$$
\begin{aligned}
& \sum_{n=0}^{\infty}\left[\left(x^{2}+2\right) h_{n}\left(p_{1},\left[-p_{2}\right]\right)-x h_{n+1}\left(p_{1},\left[-p_{2}\right]\right)\right] t^{n} \\
& =\frac{2-x t}{1-x t-t^{2}}
\end{aligned}
$$

Thus we get the following theorem.

Theorem 6. We have the following a generating function of the Lucas polynomials as

$$
\sum_{n=0}^{\infty} L_{n}(x) t^{n}=\frac{2-x t}{1-x t-t^{2}} .
$$

Proof. The ordinary generating function associated is defined by

$$
G\left(L_{n}(x), t\right)=\sum_{n=0}^{\infty} L_{n}(x) t^{n} .
$$

Using the initial conditions, we get

$$
\begin{aligned}
\sum_{n=0}^{\infty} L_{n}(x) t^{n} & =L_{0}(x) t^{0}+L_{1}(x) t+\sum_{n=2}^{\infty} L_{n}(x) t^{n} \\
& =2+x t+\sum_{n=2}^{\infty}\left(x L_{n-1}(x)+L_{n-2}(x)\right) t^{n} \\
& =2+x t+x \sum_{n=2}^{\infty} L_{n-1}(x) t^{n}+\sum_{n=2}^{\infty} L_{n-2}(x) t^{n} .
\end{aligned}
$$

Consider that $j=n-2$ and $p=n-1$. Then can be written by

$$
\begin{aligned}
& =2+x t+x\left(\sum_{p=1}^{\infty} L_{p}(x) t^{p+1}\right)+t^{2} \sum_{j=0}^{\infty} L_{j}(x) t^{j} \\
& =2+x t+x t\left(\sum_{p=0}^{\infty} L_{p}(x) t^{p}-L_{0}(x)\right)+t^{2} \sum_{j=0}^{\infty} L_{j}(x) t^{j} \\
& =2+x t+x t \sum_{p=0}^{\infty} L_{p}(x) t^{p}-2 x t+t^{2} \sum_{j=0}^{\infty} L_{j}(x) t^{j},
\end{aligned}
$$

which is equivalent to

$$
\begin{gathered}
\left(1-x t-t^{2}\right) \sum_{n=0}^{\infty} L_{n}(x) t^{n}=2-x t \\
\Rightarrow \sum_{n=0}^{\infty} L_{n}(x) t^{n}=\frac{2-x t}{1-x t-t^{2}} .
\end{gathered}
$$

This completes the proof.

Replacing $t$ by $(-t)$ in (3.8) and (3.10), we have the following theorems.

Theorem 7. We have the following a new generating function of the Fibonacci polynomials at negative coefficient as

$$
\sum_{n=0}^{\infty} F_{n}(-x) t^{n}=\frac{1}{1+x t-t^{2}} .
$$

Theorem 8. We have the following a new generating function of the Lucas polynomials at negative coefficient as

$$
\sum_{n=0}^{\infty} L_{n}(-x) t^{n}=\frac{2+x t}{1+x t-t^{2}} .
$$

\section{Conclusion}

In this paper, a new theorem has been proposed in order to determine the generating functions. The proposed theorem is based on the symmetric functions. The obtained results agree with the results obtained in some previous works. 


\section{Acknowledgments}

The authors would like to thank the anonymous referees for their valuable comments and suggestions.

\section{References}

[1] A. Abderrezzak, Généralisation de la transformation d'Euler d'une série formelle, Adv. Math. 103, 1994, 180-195.

[2] A. Abderrezzak, M. Kerada, A. Boussayoud, Generalization of Some Hadamard Product, Commun. Appl. Anal. 20(3), 301-306, (2016).

[3] A. Boussayoud, M. Boulyer, M. Kerada, On Some Identities and Symmetric Functions for Lucas and Pell Numbers, Electron .J.Math. AnalysisAppl. 5, 2017, 202-207.

[4] A. Boussayoud, N. Harrouche, Complete Symmetric Functions and $k$ - Fibonacci Numbers, Commun. Appl. Anal. 20(4), 2016, 457-467.

[5] A. Boussayoud, M. Kerada, M. Boulyer, A simple and accurate method for determination of some generalized sequence of numbers, Int. J. Pure Appl. Math.108, 2016, 503-511.
[6] A. Boussayoud, A. Abderrezzak, M. Kerada, Some applications of symmetric functions, Integers. 15, A\#48, 2015, 1-7.

[7] A. Boussayoud, M. Kerada, A. Abderrezzak, A Generalization of Some Orthogonal Polynomials, Springer Proc. Math. Stat. 41, 2013, 229-235.

[8] M. Merca, A Generalization of the symmetry between complete and elementary symmetric functions, Indian J. Pure Appl. Math. 45, 2014, 75-89.

[9] S. Falcon, A. Plaza, On the Fibonacci $k$ - numbers, Chaos, Sulutions \& Fractals. 32, 2007, 1615-1624.

[10] S. Falcon, On the $k$-Lucas Numbers of Arithmetic Indexes, Appl. Math. 3, 2012, 1202-1206.

[11] S. Falcon, On the k-Lucas numbers, J.Math.Comput.Sci. 2, 2012, 425-434.

[12] S. Falcon, On the complex k-Fibonacci numbers, Cogent Math. 3, 2016, 1-9.

[13] T. Koshy, Fibonacci and Lucas Numbers with Applications, Wiley-Interscience, 2001.

[14] Y. K.Gupta, V. H. Badshah, M. Singh, K. Sisodiya, Some Identities of Tribonacci Polynomials, Turkish Journal of Analysis and Number Theory. 4, 2016, 20-22.

[15] Y. K.Gupta, V. H. Badshah, M. Singh, K. Sisodiya, Diagonal Function of k-Lucas Polynomials, Turkish Journal of Analysis and Number Theory. 3, 2015, 49-52. 